\title{
The tuning of a small four-stroke spark ignition engine for flexible valve timings through numerical approach
}

\author{
M. Haziq Adham Rosli ${ }^{1}$, M. Razali Hanipah ${ }^{1,2^{*}}$, and Maurice Kettner $^{3}$ \\ ${ }^{1}$ Automotive Engineering Research Group (AERG), Faculty of Mechanical Engineering, Universiti Malaysia Pahang, \\ ${ }^{2}$ Automotive Engineering Centre (AEC), Universiti Malaysia Pahang, 26600 Pekan, Pahang, Malaysia \\ ${ }^{3}$ Karlsruhe University of Applied Sciences (HsKA), 76133 Karlsruhe, Germany
}

\begin{abstract}
Variable valve timing has been implemented by various manufacturers to improve internal combustion engine performance while operating at wide speed and load ranges. A novel flexible valve timing system for a small four-stroke engine is currently under development by Automotive Engineering Research Group (AERG) in Universiti Malaysia Pahang (UMP). In this paper, a comprehensive intake and exhaust tuning for the flexible variable valve timing is presented. A numerical assessment has been conducted through one dimensional engine modelling and simulation using validated model. There are eight valve timing configurations investigated for the tuning for three main speed regions. The simulation shows a positive and significant impact to the engine performance in three approaches; namely late intake valve closing, early intake valve closing and late exhaust valve closing. These approaches sufficiently covered the whole range of engine speeds for optimum engine operational performance.
\end{abstract}

\section{Introduction}

The emissions regulation standards have been continuously improved due to ever increasing environmental concern. It drives automotive manufacturers to produce cars that meet those standards. In addition, internal combustion engines role as road vehicles' prime mover are diminishing as the demand on electric powered automobiles has increased significantly. Thus, various means of improvement have been devised over the years in the area of internal combustion engines. Variable valve timing (VVT) has been identified as one of the crucial technology to enhance internal combustion engine performance. VVT offers an additional control so that the engine operating conditions may be tailored more precisely hence output and performance were amplified [1]. The flexibility of the valve timing adjustment, duration, lift, or a combination of these could possibly result an improvement to the performance, fuel economy and emissions of the SI engines [2, 3], improved engine braking, exhaust residual control, and cylinder deactivation [4].

Late Intake Valve Closing (LIVC) strategy on SI single cylinder engine with three different cams was presented by Tuttle [5] in which one per cent loss in torque from the maximum best torque in part-load was reported. According to Rabia S. M. and Kora N. S. [6] , knocking in LIVC engines was found to increase with decreasing engine speed. This is due to LIVC demands more spark advance compared to conventional engine especially at part-load. LIVC strategy present difficulties because early ignition can occur before intake valve has been closed at very low loads [7].

Asmus [8] explained that volumetric efficiency increases by delaying the intake valve closing at higher speed with the risk of charge reverse flow. Similar observation was made by Neher [9], which resulted in poor engine efficiency due to reverse flow occurs during compression stroke that later cause loss during compression.

Sellnau and Rask [10] stated that the strategy of Early Intake Valve Closing (EIVC) demonstrated a significant improvement to low-speed BMEP and $12 \%$ volumetric efficiency improvement with low-lift cam duration. According to De Bellis, Gimelli [11] in their paper of investigating the effects of pre-lift intake valve strategies on DISI Turbocharged Engine, summarized that the prelift adoption carries the potential to bring improvement on engine performance and fuel economy at full load operations for the speeds of 2000 and $3000 \mathrm{rpm}$, without penalizing the fuel consumption. Urata, Umiyama [12] stated that EIVC lowers the gas temperature during compression stroke because there is lesser amount of charge to burn.

The Late Intake Valve Opening (LIVO) was found to create turbulence for the incoming air and fuel mixture which helps to create homogeneous combustion) [13]. Law, Kemp [14] indicated that due to Early Exhaust Valve Closing (EEVC), a small amount of pumping losses occurred due to the compression and expansion of trapped residual gas. Hong, Parvate-Patil [13] claimed that Late Exhaust Valve Opening (LEVO) causes majority of work shifts to the exhaust stroke and greater expansion work

* Corresponding author. Email: mohdrazali@ump.edu.my 
and increase the pumping loss at the beginning of exhaust stroke.

Based from wide literature review on previous works regarding the research and study of variable valve timings in internal combustion engine, eight strategies as summarised by Parvate-Patil, Hong [15] were proposed to be applied in this investigation to inspect how they affect the engine in terms of performance and efficiency.

The purpose of this paper is to illustrate the tuning of valve timings conducted using numerical approach on a small single cylinder spark ignition four-stroke cycle engine.

\section{Baseline engine specifications}

The investigation was conducted numerical model developed based on a $65 \mathrm{cc}$ four-stroke gasoline engine manufactured by Andreas Stihl AG \& Company KG, Germany. The engine stroke is $33 \mathrm{~mm}$ and the bore is $50 \mathrm{~mm}$. The geometric compression ratio is $9.5: 1$, while the maximum valve lift has been measured to be $2.74 \mathrm{~mm}$ and this engine is designed for single valve timing configuration. The valve diameter for the intake is $20 \mathrm{~mm}$ and for exhaust is $18 \mathrm{~mm}$. The engine detailed specifications can be viewed as in Table 1 .

Table 1. Details of Baseline Engine Specifications.

\begin{tabular}{|c|c|}
\hline Parameter & Value \\
\hline Capacity & $65 \mathrm{cc}$ \\
\hline Bore [mm] & 50 \\
\hline Stroke [mm] & 30 \\
\hline Geometric Compression Ratio & $9.5: 1$ \\
\hline Max. Power (@7200RPM) & 2.3 \\
\hline Maz. Valve Lift [mm] & 2.74 \\
\hline Intake Valve Diameter [mm] & 20 \\
\hline Exhaust Valve Diameter [mm] & 18 \\
\hline
\end{tabular}

\section{Methodology}

The valve timing tuning was done through onedimensional (1D) numerical analysis. The whole process is summarised in Figure 1. The first step is to divide the engine into four main subsystems. Then, relevant geometric data from manufacturer datasheet and measurements are entered into each component of all subsystems. Finally, the operating parameters of the engine are defined. The final output of the numerical simulation is the engine performance parameters which can be processed in various forms.

The 1D tool used in this project is GT-SUITE by Gamma Technologies while MATLAB was used in plotting the simulation results.

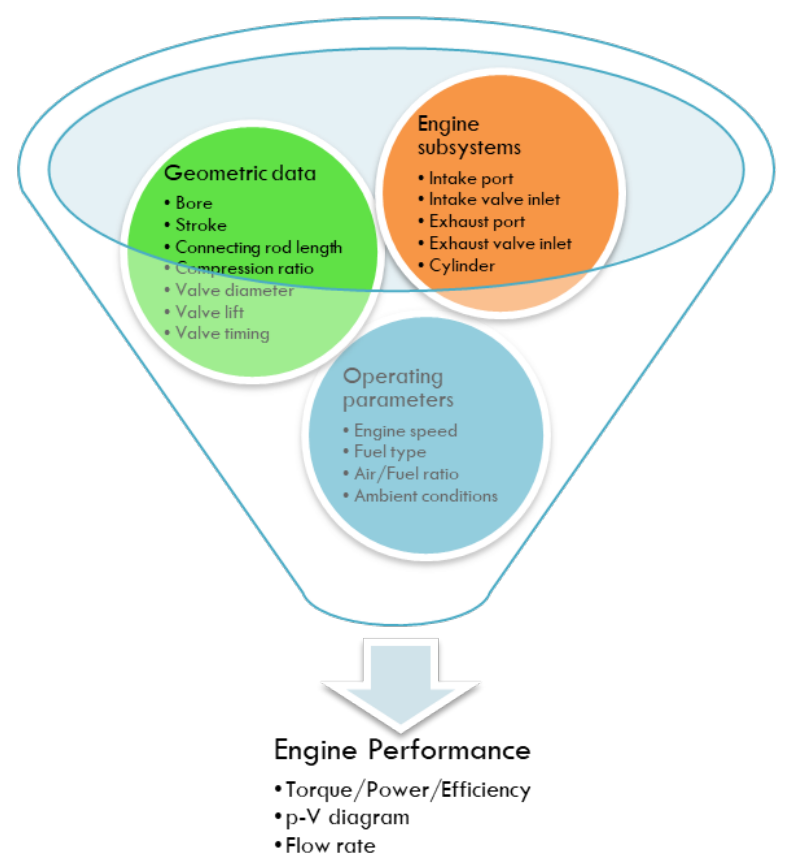

Fig. 1. The procedure of one-dimensional numerical analysis.

\subsection{One dimensional model development}

The structure of the engine which set as a baseline model in this project is as shown as in Figure 2. The injection rate of the engine model was set to be continuously adjusted at stoichiometric.

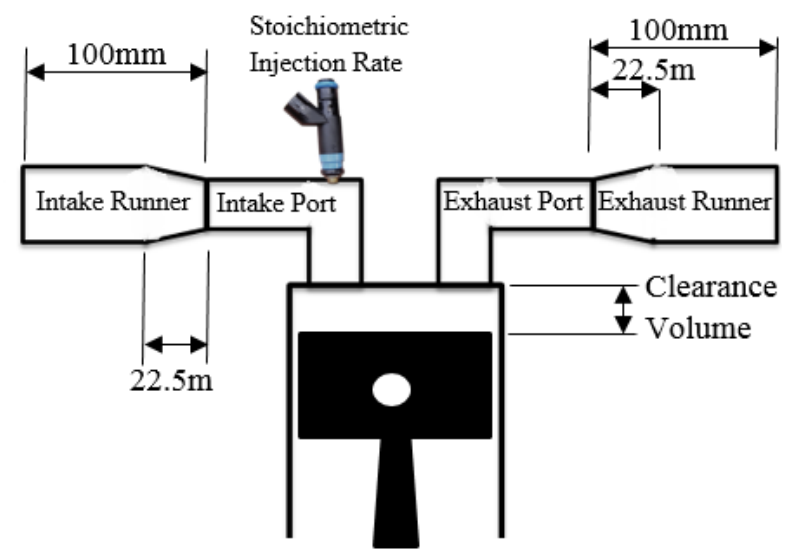

Fig. 2. Components of the baseline model.

The GT-POWER model of the engine is shown as in Figure 3.

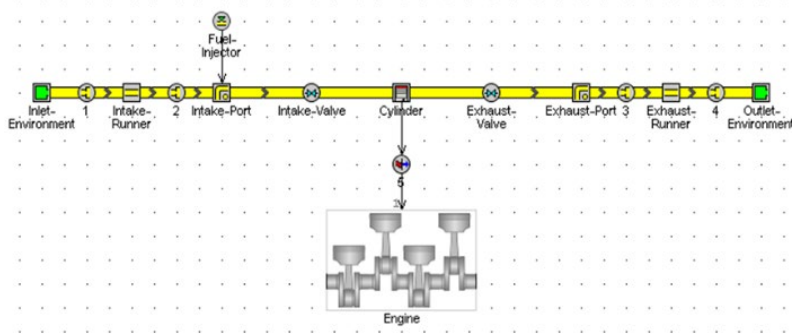

Fig. 3. One Dimensional Structure of Baseline Model in GTPOWER 
The flow coefficient values of intake and exhaust ports were taken from experimental result by Zahidi, Hanipah [16].

\subsection{Valve timing tuning}

The valve timing tuning is essential in order to obtain preliminary valve settings for various engine speeds for the flexible valve timing system. This is done by evaluating the impact of various valve timing strategies to the engine performance. The key initial step is to define the speed boundary between each engine operating regime.

In this investigation, the engine operating regions are divided into three modes which are:

- low speed (from 1,000RPM to 4,000RPM),

- medium speed (from 4,000RPM to 7,000RPM), and

- $\quad$ high speed (from 7,000RPM to 10,000RPM).

The eight strategies tuning philosophies is illustrated in Figure 4. Each curve of the baseline valve timing is adjusted by 10 degrees of crank angle $\left({ }^{\circ} \mathrm{CA}\right)$, in advancing and retarding trends.

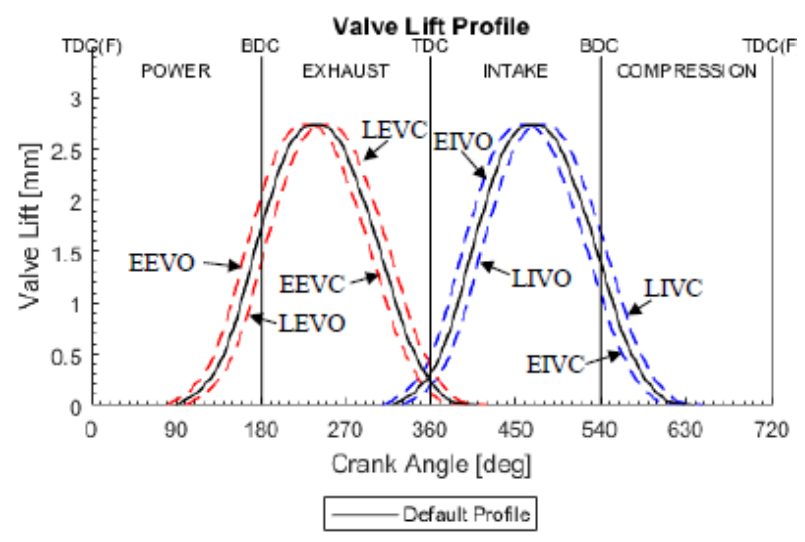

Fig. 4. Flexible Valve Timing Strategies

\section{Model validation}

The peak power for the original $65 \mathrm{cc}$ Stihl $4 \mathrm{MIX}$ is $2.3 \mathrm{~kW}$ at $7200 \mathrm{rpm}$ as acquired from manufacturer data which can be set as a reference to validate the baseline simulation model. The model was validated using the peak power specification from manufacturer and was compared with the fitted power curve (in polynomial equation) from the Ricardo WAVE simulation by Hanipah [17].

Figure 5 shows the brake power of the developed model compared with model built in previous study using Ricardo WAVE Simulation software. The maximum power produced is $2.3 \mathrm{~kW}$ at $7000 \mathrm{RPM}$ which shows acceptable correlation to the fitted power curve from 4000RPM and above with error of percentage of less than $20 \%$.

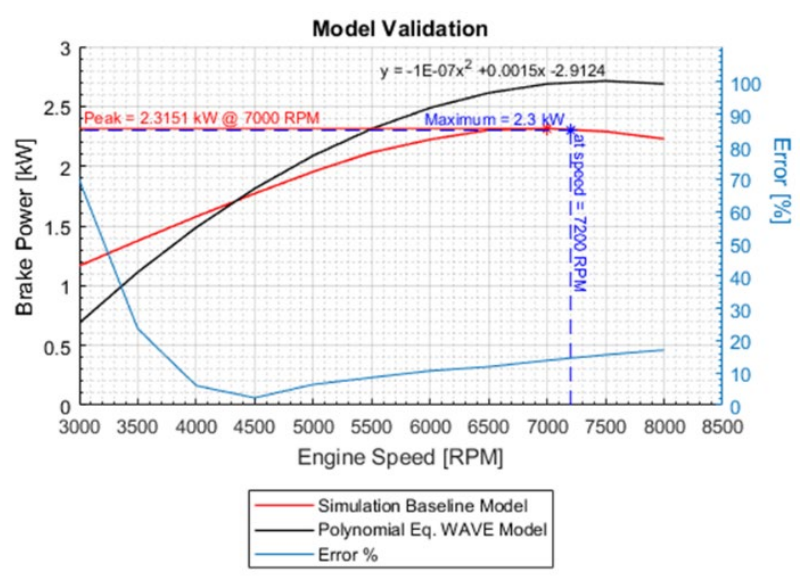

Fig. 5. Power Curve Validation from GT-POWER Simulation

This accuracy acceptable for the purpose outlined in this paper. It is to be improved in the future with further results from dynamometer testing of the engine.

\subsection{CA50 Tuning}

The position where the accumulated heat release reaches $50 \%$ of the total released heat is called CA50 which is the parameter that is optimised to obtain a validated baseline model in the simulation. The CA50 serves as ignition point to control the work output of the engine model. CA50 is the location is based on the SI Wiebe combustion model curve. Wiebe function used in this simulation can be expressed as:

$$
x_{b}=1-\exp \left[-a\left(\frac{\theta-\theta_{0}}{\Delta \theta}\right)^{m+1}\right]
$$

Where $\theta$ is crank angle degrees with $\theta_{0}$ corresponding to the initialization of heat release and $\Delta \theta$ corresponding to the duration of burn.

Narrowing the timing result a higher maximum power produced, while widening the timing result a lower maximum power produced. Indirectly, the adjustment of CA50 is associated with varying the ignition timing of the engine.

\section{Results and discussions}

\subsection{The impact of late intake valve closing (LIVC)}

Figure 6 and Figure 7 show the brake mean effective pressure (BMEP) and volumetric efficiency from the late intake valve closing (LIVC) strategy respectively. It was observed that LIVC yield performance improvement at high-speed region. 


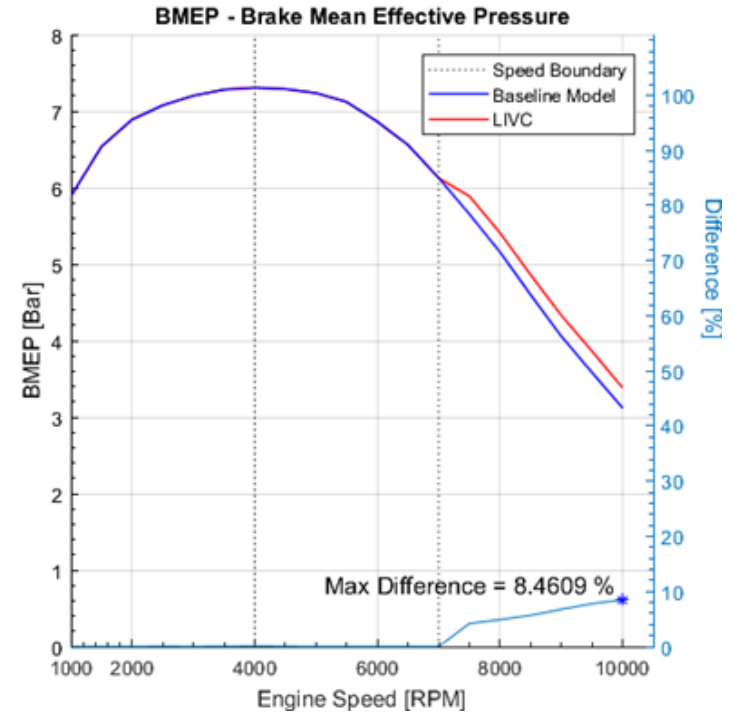

Fig. 6. BMEP curve of LIVC at high speed.

The curve starting to show an increase when it reaches around 6000RPM during tested in medium speed mode and keep showing a performance inclination until entering high speed mode. In high engine speed mode, the LIVC strategy clearly shows a positive impact to the engine performance in terms of Brake Mean Effective Pressure with maximum percentage of improvement up to $8.4 \%$ and volumetric efficiency with maximum percentage of $6.1 \%$.

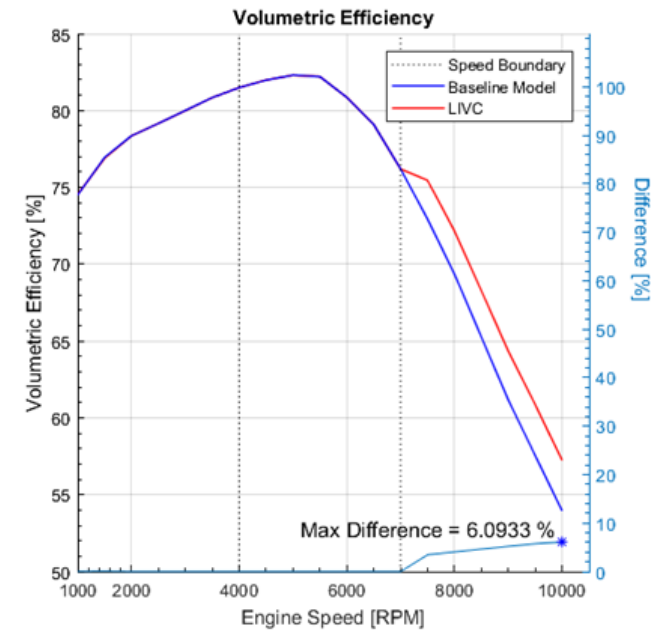

Fig. 7. Volumetric Efficiency curve of LIVC at high speed.

On the other hand, LIVC has resulted in pressure loss during power stroke at low speed as observed in Figure 8. The starting of compression produces lower pressure due to late closing of intake valve, thus less pressure produce at the end of power stroke and less net amount of work produced by compression stroke.

In medium speed mode, ram effect was observed to take place, however, pressure loss still occurs because of the low momentum of the mixture flowing in to charge the cylinder to avoid reverse flow. In high speed mode, a great ram effect was observed to happen and because of high engine speed, the high momentum of air and fuel mixture continue to charge the cylinder resulting a higher pressure produced at the end of compression stroke and higher net amount of work produced by the compression stroke.

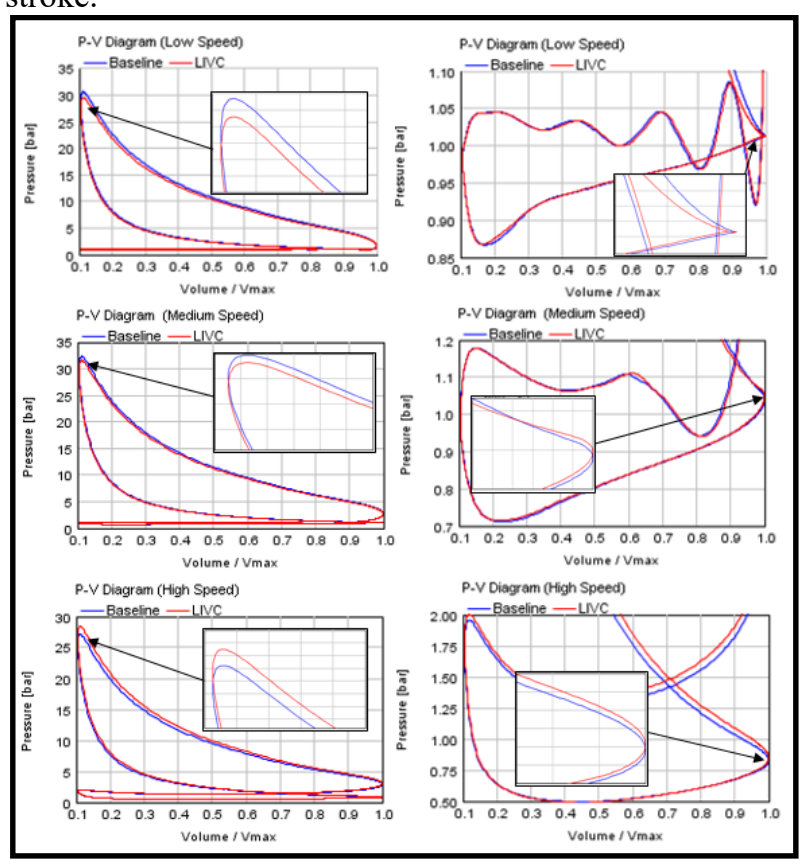

Fig. 8. p-V Diagram of LIVC strategy in 3 speed mode. (Each graph on right column represents the emphasis on the pumping work of each of the $\mathrm{p}-\mathrm{V}$ diagram on the left column.)

In addition, LIVC strategy was observed to reduce little amount of pumping work in medium and high speed modes.

Dresner and Barkan [18] concluded that late intake valve closing cannot yield a great improvement in fuel economy in lower speed region because of high flow losses.

\subsection{The impact of early intake valve closing (EIVC)}

Figure 9 and Figure 10 show the brake mean effective pressure (BMEP) and volumetric efficiency from the EIVC strategy respectively.

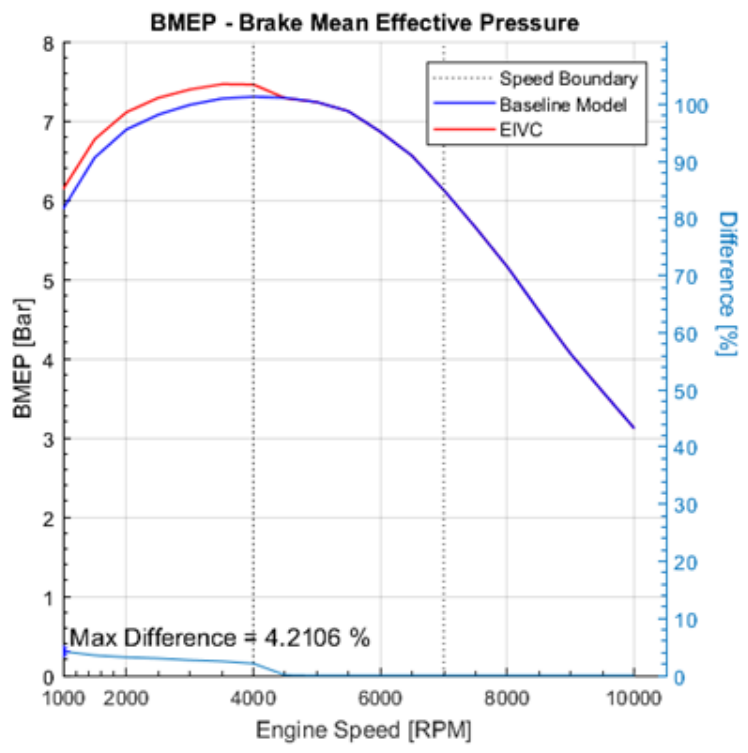

Fig. 10. BMEP curve of EIVC at low speed. 
Effect of EIVC yield a similar result in terms of volumetric efficiency where there is maximum percentage of improvement of $3.9 \%$ during low speed or idle state. The curve continue showing imrpovement up until medium speed.

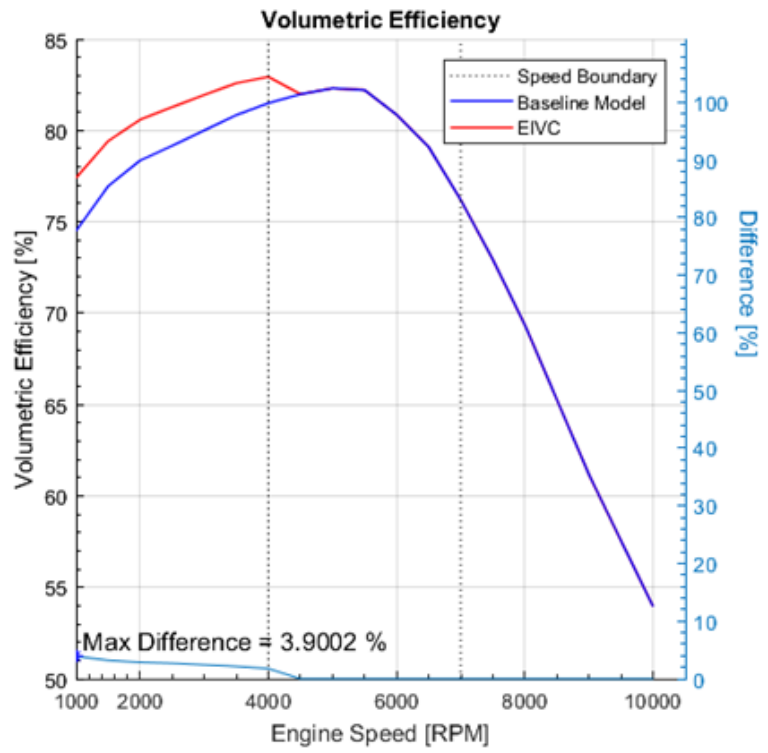

Fig. 10. Volumetric Efficiency curve of EIVC at low speed.

$\mathrm{Li}$, Tao [19] found that with the IVC timing being changed earlier, the output torque curve would move to left side compared to the baseline. The most interesting observation is the incremental of increase in output torque at low engine speed.

As observed in Figure 11, in low speed mode, EIVC has resulted a higher pressure in the beginning of compression stroke. This is due to early closing of intake valve causing the cylinder to be isolated, which reduces amount of air and fuel inducted, and not causing reverse flow to the intake manifold during compression stroke, thus increasing the efficiency and higher pressure created. The performance starting to deteriorate in medium speed mode due to the yearning of charge is limited by the early closing of intake valve. In medium and high speed mode, more mass of air and fuel mixture needed in order to create a higher power. The early closing of intake valve prevent more amount of charge inducted in the cylinder, lead to insufficient amount of charge to burn to create necessary combustion to produce higher power.

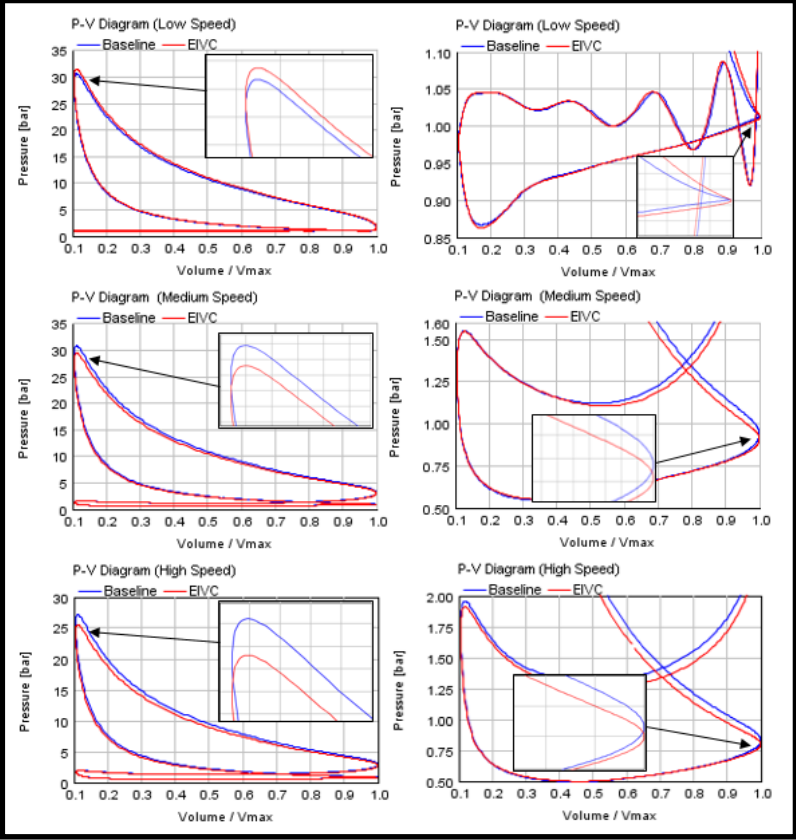

Fig. 11. p-V Diagram of EIVC strategy in 3 speed mode. (Each graph on right column represents the emphasis on the pumping work of each of the $\mathrm{p}-\mathrm{V}$ diagram on the left column.)

\subsection{The impact of late intake valve opening (LIVO)}

Figure 12 shows the brake mean effective pressure curve when LIVO strategy was applied in high speed mode.

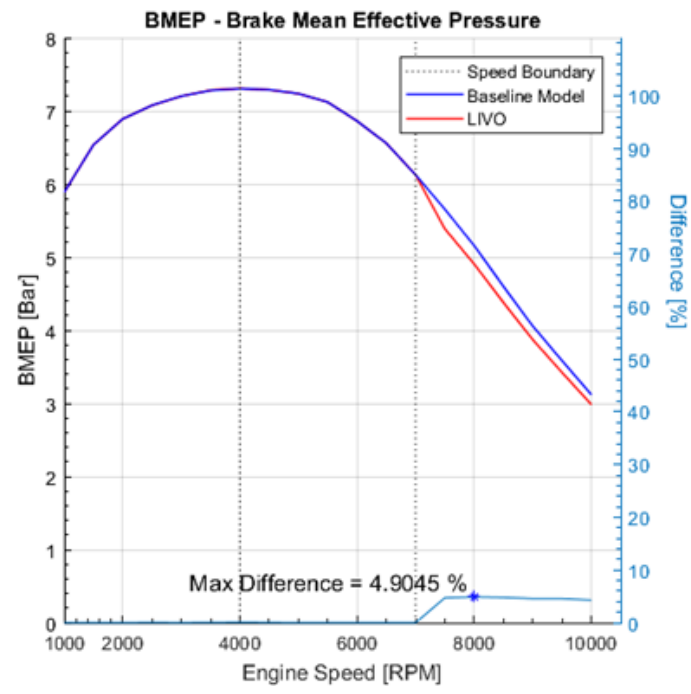

Fig. 12. BMEP curve of EIVC at low speed.

From the engine performance results, Late Intake Valve Opening (LIVO) strategy brings no positive impact to the engine in terms of performance in all speed levels. In high engine speed mode, the late intake valve opening strategy clearly shows a negative impact to the engine performance in terms of brake mean effective pressure and brake power with maximum percentage of depletion up to $4.9 \%$.

As observed in Figure 13, in low speed mode, late intake valve opening (LIVO) strategy has resulted a significant addition to the pumping loss where more 
energy required to perform pumping work. This is due to the vacuum effect occur in the combustion chamber because of the late opening of the intake valve to allow smooth flow after exhaust stroke ends. The rest of the curve shows no significant different throughout the complete cycle. In medium and high speed mode, a greater amount of pumping loss was observed, resulting lower pressure produced at the end of compression stroke.

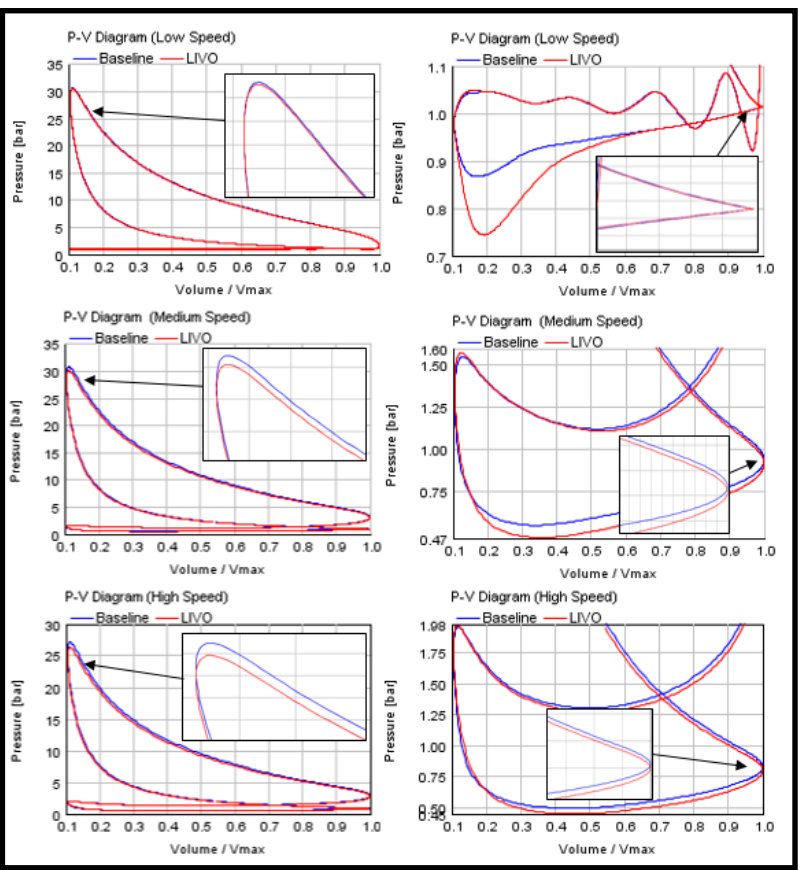

Fig. 13. p-V Diagram of LIVO strategy for all three speed regions (Each graph on right column represents the emphasis on the pumping work of each of the $\mathrm{p}-\mathrm{V}$ diagram on the left column).

\subsection{The impact of early intake valve opening (EIVO)}

As can be observed in Figure 14, EIVO has resulted a significant reduction of pumping loss where less energy required to perform pumping work. In low speed mode, the pumping work reduced significantly at the beginning of the intake stroke thus eliminate some pumping loss. This is due to the early opening of the intake valve which provide continuous smooth flow during the suction of airfuel charge.

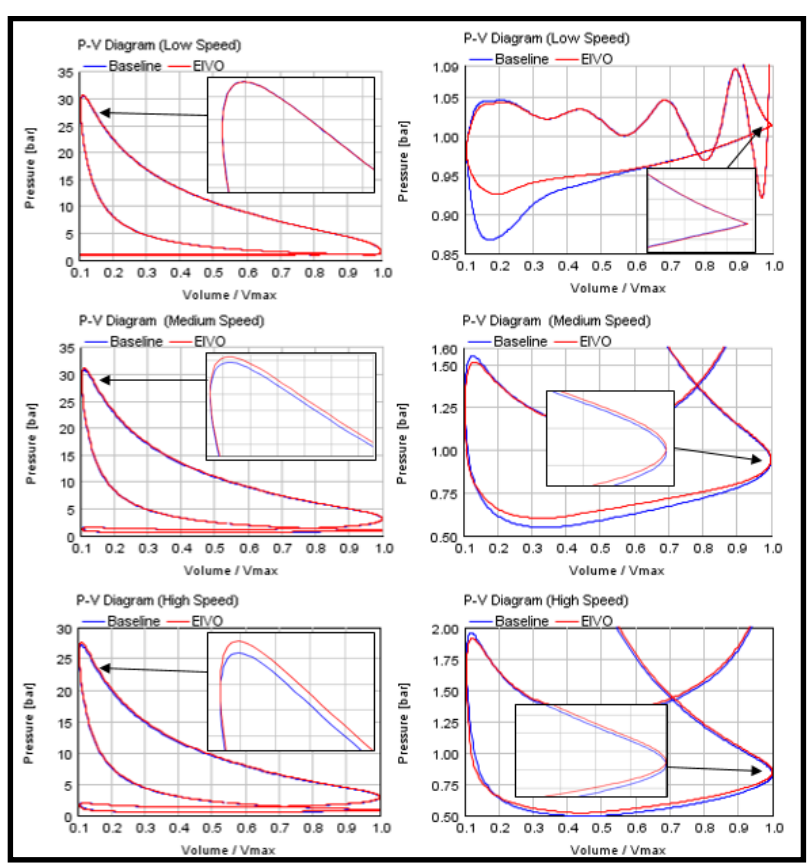

Fig. 14. p-V Diagram of EIVO strategy in 3 speed mode. (Each graph on right column represents the emphasis on the pumping work of each of the $\mathrm{p}-\mathrm{V}$ diagram on the left column.)

This is in contrast to the LIVO strategy which limit the flow in the beginning of intake stroke. The rest of the curve shows no significant different throughout the complete cycle. In medium speed and high speed mode, a lesser amount of pumping loss produced. In addition, small ram effect was observed resulting higher pressure produced at the end of compression stroke. Higher pressure in the beginning of the power stroke, resulting larger amount of work done.

\subsection{The impact of late exhaust valve closing (LEVC)}

Figure 15 and Figure 16 show the brake mean effective pressure (BMEP) and volumetric efficiency from the LEVC strategy respectively. LEVC strategy evidently prove negative effect to the engine performance in low speed mode. In high engine speed mode, the LEVC strategy enhance the engine performance in terms of brake mean effective pressure with maximum percentage of augmentation up to $12.74 \%$ 


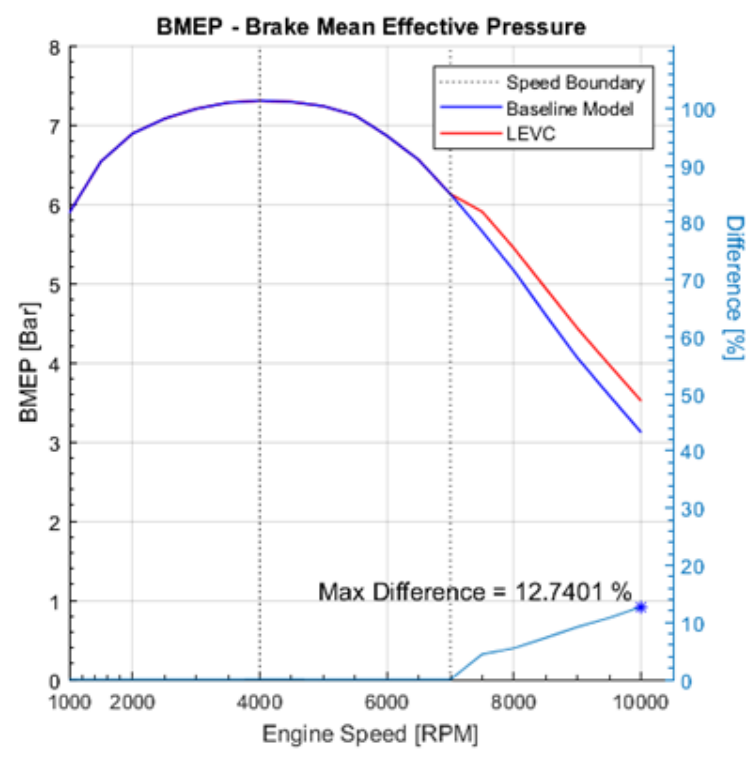

Fig. 15. BMEP curve of LEVC at high speed.

The impact on the volumetric efficiency in LEVC strategy follow the same trend of its impact on the BMEP. When LEVC strategy is applied in low engine speed mode, maximum reduction with small percentage value of $1.06 \%$ in volumetric efficiency compared with baseline model. When LEVC strategy is applied in medium speed mode, the efficiency curve shows slight improvement with a maximum difference in volumetric efficiency of $1.8 \%$ and remain positive until the end of the speed mode. While in high engine speed mode, the LEVC strategy clearly shows a positive impact to the engine efficiency with maximum percentage of improvement up $7.00 \%$.

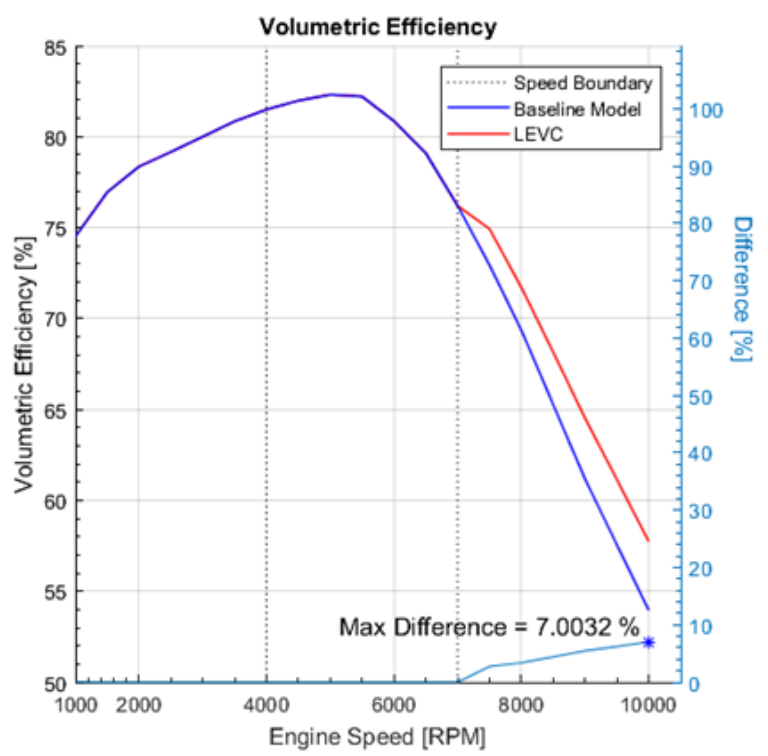

Fig. 16. Volumetric Efficiency curve of LEVC at high speed.

From the volumetric efficiency result of LEVC strategy application, it can be concluded in overall that late exhaust valve closing (LEVC) strategy carry a positive impact to the engine in terms of efficiency in medium and high speed mode.
As observed in Figure 17, LEVC has resulted quite significant reduction of pumping loss where less energy required to perform pumping work. This can be observed in all speed mode at the point of exhaust valve closing or end of exhaust stroke. The reason of this matter is in LEVC, the period of valve overlap increases due retardation of exhaust valve closing point (exhaust valve duration increase). Some of the burnt gases from the exhaust manifold own possibility to flow back into the intake manifold during induction stroke and reduces the pumping loss. During the intake of new charge, the intake manifold pressure is high, the short duration of exhaust valve opening slightly help the flow occurrence in the cylinder chamber.

As speed increase, the reduction of pumping loss is significantly shown with pressure value at the end of exhaust stroke minimized. LEVC really help in reducing the pumping loss as engine gains its speed. In medium and high speed, the maximum pressure produced in the end of compression stroke are higher compared to the baseline. Lesser amount of pumping work produced resulting higher pressure produced at the end of compression stroke [13]. This factor explains the improvement of the engine performance in terms of brake mean effective pressure (BMEP) in medium and high speed mode.

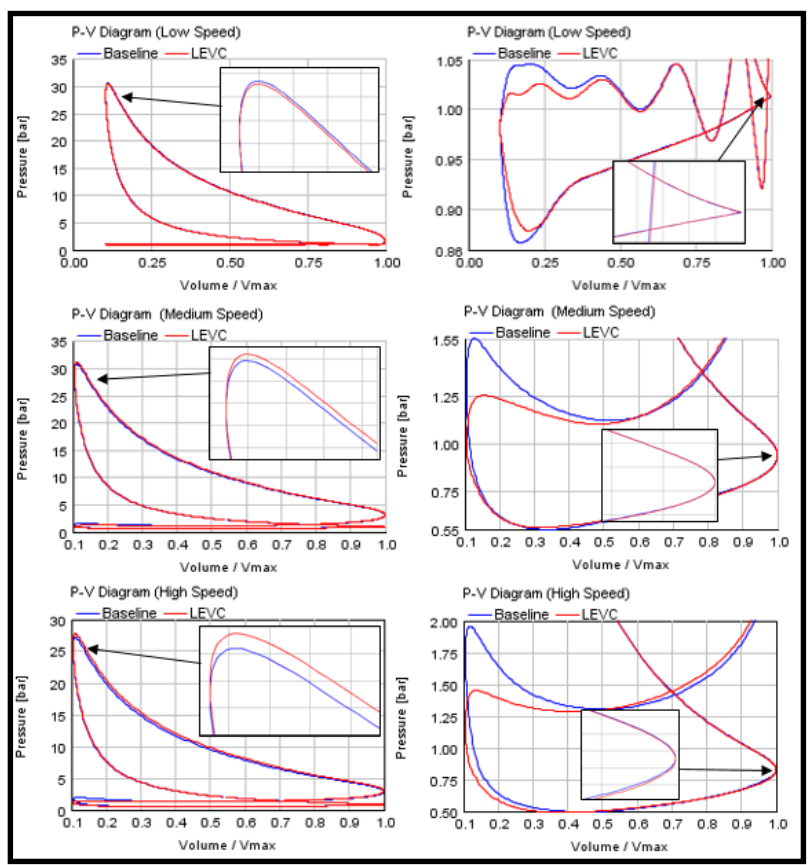

Fig. 17. p-V Diagram of LEVC strategy in 3 speed mode. (Each graph on right column represents the emphasis on the pumping work of each of the $\mathrm{p}-\mathrm{V}$ diagram on the left column.)

\subsection{The impact of early exhaust valve closing (EEVC)}

Based on Figure 18, EEVC has resulted a great addition to the pumping loss where more energy required to perform pumping work in all speed mode. This is due to less valve overlap duration with a shorter period of exhaust valve opening. The early closing of exhaust valve cause the exhaust gases not completely drawn out from the cylinder and not fully release the pressure from 
combustion. The partial of exhaust gases not expelled and trapped inside the cylinder. This flow restriction causes high pressure in the cylinder produced in the end of exhaust stroke, thus increase the pumping work for the engine. As engine speed increasing, the pressure at the end of exhaust stroke becomes higher thus a greater amount of pumping loss created. This leads to lower maximum pressure produced at the end of compression stroke as speed of the engine increasing. However, in low speed mode, the maximum pressure produced in the end of compression stroke is not affected as observed in the pV diagram.

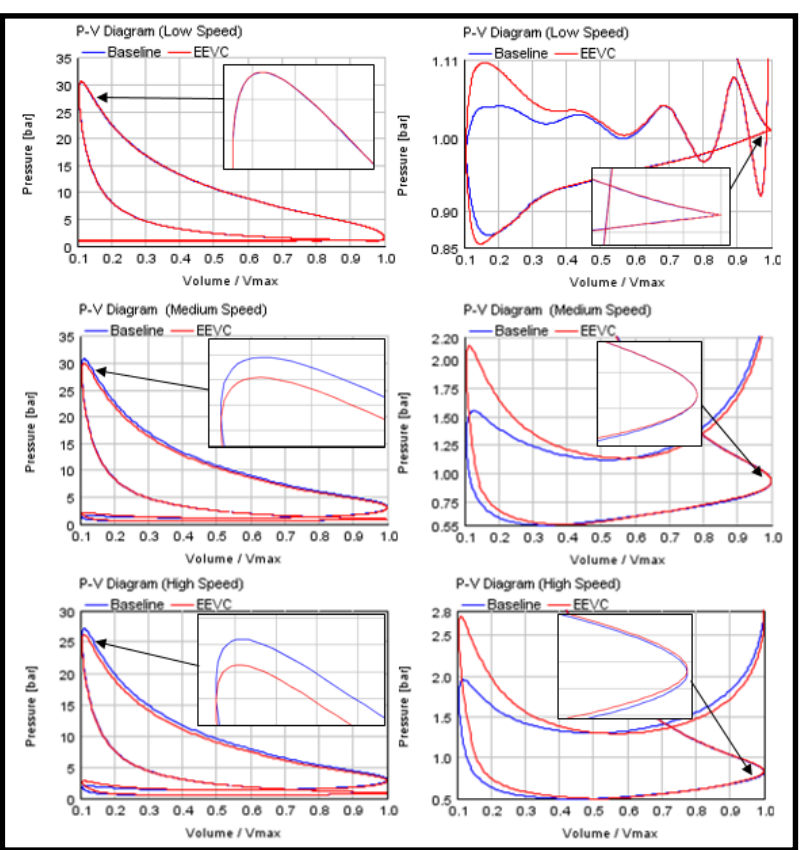

Fig. 18. p-V Diagram of EEVC strategy in 3 speed mode. (Each graph on right column represents the emphasis on the pumping work of each of the $\mathrm{p}-\mathrm{V}$ diagram on the left column.)

\subsection{The impact of late exhaust valve opening (LEVO)}

With reference to Figure 19, the variation of p-V Diagram curve of late exhaust valve opening (LEVO) strategy can be observed in three different speed mode application. In low speed mode, LEVO has resulted different curve of exhaust stroke showing the delay of pressure released from the cylinder. This is due to late exhaust valve opening which restricted the combustion residue to be expelled early and increase the pumping work. As speed increases, the pumping work was observed to be greater resulting lower maximum pressure produced at the end of compression stroke and higher pressure in the cylinder during exhaust stroke. LEVO strategy reduces the power output because most of the work is from exhaust stroke which is used to expel combustion product.

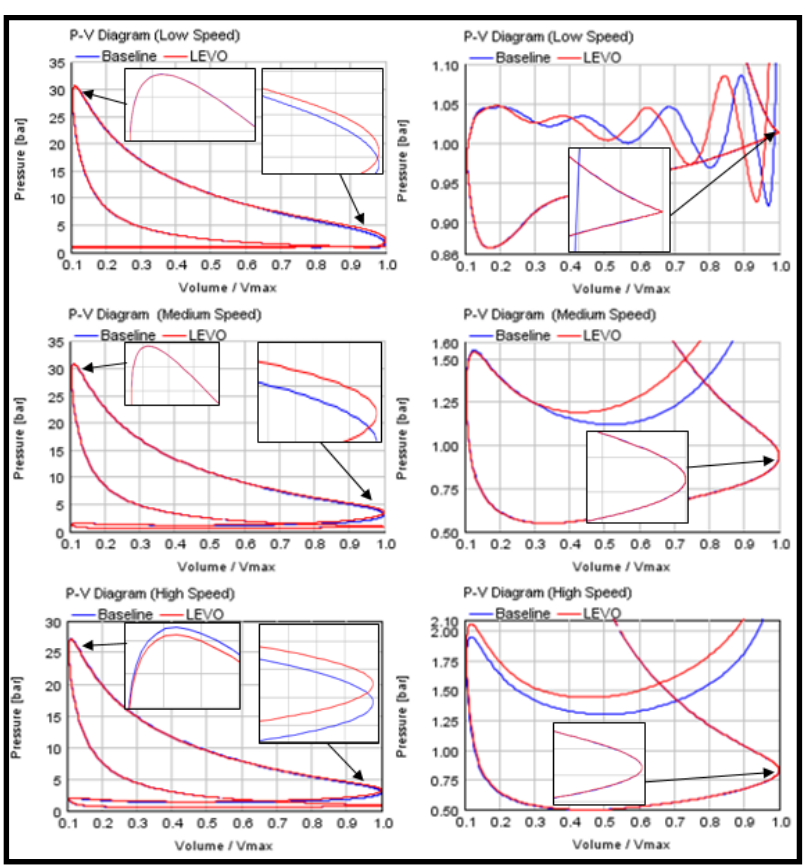

Fig. 19. $p-V$ Diagram of LEVO strategy in 3 speed mode. (Each graph on right column represents the emphasis on the pumping work of each of the $\mathrm{p}-\mathrm{V}$ diagram on the left column.)

\subsection{The impact of early exhaust valve opening (EEVO)}

With reference to Figure 20, in low speed mode, EEVO has resulted different curve of exhaust stroke showing the advancement of pressure released from the cylinder. This is due to early exhaust valve opening which allow the combustion residue to be expelled early and decrease the pumping work during exhaust as speed increases. However, this phenomenon is identified as blowdown loss where the pressure was release early during the power stroke, resulting less work produced.

In the high speed mode, the pumping work was observed to be lower resulting slightly higher maximum pressure produced at the end of compression stroke. However, this percentage is very low and insignificant. The large impact can only be observed when the engine is running at ultimately higher speed. This strategy is possible to be implemented in F1 engine as the engine running at distinctly higher RPM than the typical stock engine. Early exhaust valve opening (EEVO) strategy produce lower pressure in the cylinder during exhaust stroke. This impact can be observed at the starting of exhaust stroke curve. EEVO provides better scavenging of burned gases, but it causes a reduction in the expansion work (power stroke blowdown loss), thus reduces the output power of the engine. 


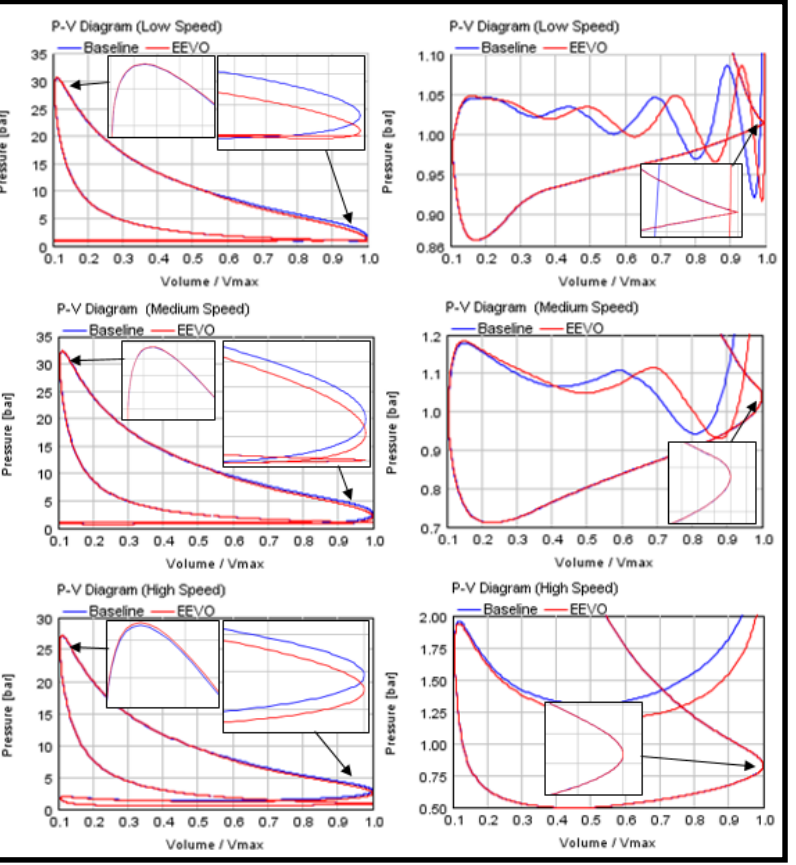

Fig. 20. p-V Diagram of EEVO strategy in 3 speed mode. (Each graph on right column represents the emphasis on the pumping work of each of the $\mathrm{p}-\mathrm{V}$ diagram on the left column.)

\section{Conclusions}

In summary, Late Intake Valve Closing (LIVC) result a positive impact to the engine in terms of performance and efficiency at high speed. LIVC allows reverse flow to occur at low engine speed and is bad for the engine, while in high speed, high momentum of air and fuel charge flowing into engine cylinder causing a higher pressure produced at the end of compression stroke.

Early Intake Valve Closing (EIVC) result a positive impact to the engine in terms of performance and efficiency at low and medium speed. At low speed, EIVC cause cylinder to be isolated earlier and minimize the charge inducted, thus increasing the efficiency and perform internal cooling. However, at high speed, EIVC prevent more amount of charge to be inducted into the cylinder, lead to insufficient amount of charge to burn to create higher power.

The positive impact of 8 VVT strategies conducted in this project were summarised and classified according to the engine speed as shown as in Figure 21.

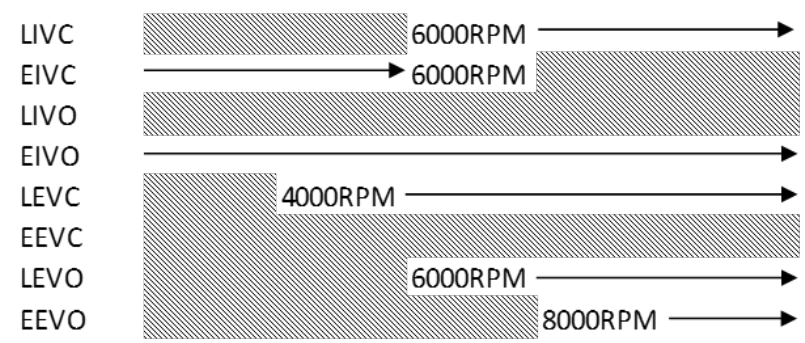

Fig. 21. Summary of Positive Impact of Flexible Valve Timing on Engine Performance.

These results can be used for developing the programming of the flexible valve timing controller in the next stage.
In conclusion, a positive and significant impact to the engine performance can be achieved using 3 approaches; LIVC, EIVC and LEVC individually. Most positive impact produced from varying the intake valve timing. In addition, retarding the exhaust valve closing timing also produced a positive impact.

The authors would like to thank the Ministry of Science, Technology and Innovation Malaysia (MOSTI) for the financial grant (project no. 03-01-16-SF0172/RDU170501) and also Universiti Malaysia Pahang (www.ump.edu.my) for providing laboratory facilities.

\section{References}

1. Ma, T., Effect of variable engine valve timing on fuel economy. SAE transactions, 1988: p. 665672 .

2. Turner, J., S. Kenchington, and D. Stretch. Production AVT development Lotus and Eaton's electrohydraulic closed-loop fully variable valve train system. in 25th Vienna Motor Symposium, 2004. 2004. University of Bath.

3. Lee, J. and H.J. Chang. Multi-parametric model predictive control for variable valve timing. in 2017 17th International Conference on Control, Automation and Systems (ICCAS). 2017.

4. Halderman, J.D. and C.D. Mitchell, Automotive technology. 2014: Pearson.

5. Tuttle, J.H., Controlling engine load by means of late intake-valve closing. 1980, SAE Technical Paper.

6. Rabia, S.M. and N.S. Kora, Knocking Phenomenon in Gasoline with Late-Intake Valve Closing. 1992, SAE Technical Paper.

7. Ahmad, T. and M. Theobald, A survey of variable-valve-actuation technology. SAE 891674, 1989.

8. Asmus, T.W., Valve events and engine operation. 1982, SAE Technical Paper.

9. Neher, D., Miller Cycle and Exhaust Gas Recirculation for a Naturally Aspirated Leanburn Gas Engine. 2017, Universidad de Valladolid. p. 172.

10. Sellnau, M. and E. Rask, Two-step variable valve actuation for fuel economy, emissions, and performance. 2003, SAE Technical Paper.

11. De Bellis, V., A. Gimelli, and M. Muccillo, Effects of pre-lift intake valve strategies on the performance of a DISI VVA turbocharged engine at part and full load operation. Energy Procedia, 2015. 81: p. 874-882. 
12. Urata, Y., et al., A study of vehicle equipped with non-throttling SI engine with early intake valve closing mechanism. 1993, SAE Technical Paper.

13. Hong, H., G. Parvate-Patil, and B. Gordon, Review and analysis of variable valve timing strategies - eight ways to approach. Proceedings of the Institution of Mechanical Engineers, Part D: Journal of Automobile Engineering, 2004. 218(10): p. 1179-1200.

14. Law, D., et al., Controlled combustion in an ICengine with a fully variable valve train. 2001, SAE Technical Paper.

15. Parvate-Patil, G.B., H. Hong, and B. Gordon, An Assessment of Intake and Exhaust Philosophies for Variable Valve Timing. 2003, SAE International.

16. Zahidi, M.K., et al., The two-stroke poppet valve engine. Part 1: Intake and exhaust ports flow experimental assessments. IOP Conference Series: Materials Science and Engineering, 2017. 257(1): p. 012023.

17. Hanipah, M.R.B., Development of a spark ignition free-piston engine generator. 2015, University of Newcastle upon Tyne.

18. Dresner, T. and P. Barkan, A review of variable valve timing benefits and modes of operation. 1989, SAE Technical Paper.

19. Li, L., et al., Effects of intake valve closing timing on gasoline engine performance and emissions. 2001, SAE Technical Paper. 